www.jmscr.igmpublication.org

Index Copernicus Value: 79.54

ISSN (e)-2347-176x ISSN (p) 2455-0450

crossref DOI: https://dx.doi.org/10.18535/jmscr/v7i6.43

Journal Of Medical Science And Clinical Research

IGM Publication

An Official Publication of IGM Publication

\title{
Leriche Syndrome -A Case with an Unusual Presentation
}

\author{
Authors \\ Harharpreet Kaur ${ }^{1}$, Richa Verma ${ }^{2}$, Kiranjit Kaur ${ }^{3 *}$, Navneet Singh \\ ${ }^{1}$ Professor, Department of Medicine, MM Medical College, Kumarhatti, Solan \\ ${ }^{2}$ Assistant Professor Department of Radiology, MM Medical College, Kumarhatti Solan \\ ${ }^{3}$ Professor, Department of Biochemistry, MM Medical College, Kumarhatti, Solan \\ ${ }^{4}$ Assistant Professor Department of Orthopaedics, MM Medical College, Kumarhatti Solan \\ *Corresponding Author \\ Dr Harharpreet Kaur \\ Professor Medicine, MM Medical College, Kumarhatti, Distt. Solan, Himachal Pradesh, India
}

\begin{abstract}
Leriche syndrome is caused by an atherosclerotic occlusion of the aortoiliac arteries which produces vascular insufficiency in the pelvic region.This can produce ischemia of neural structures with symptoms of neuropathy causing the diagnosis to be overlooked especially in diabetics. Our patient was a 61 year old diabetic whose presenting symptoms were acute pain and paresthesias in the back which radiated down the right lower limb and suggestive of a degenerative disc disease. The patient did not recover with the conventional treatment for the same and was reviewed. The symptoms appeared on walking and subsided on rest. This history prompted a complete vascular evaluation which established the diagnosis. The pain due to the disc disease takes time to heal but our patient made an unexpectedly rapid recovery after medical measures to improve circulation were instituted. Presumably the oxygen tension in the nerve trunk was lowered during exercise as a simultaneous increase in the blood supply through the vasa nervorum was prevented due to the aortoilliac disease. The extensive collaterals help in normalizing life in some cases as ours who sought medical help only when the radiculopathy due to a relatively mild disc disease was precipitated by the compromised circulation.

Keywords: Leriche syndrome; Radiculopathy.
\end{abstract}

\section{Introduction}

Leriche syndrome (LS) is caused by either preocclusive stenosis or complete atherosclerotic occlusion of the infrarenal aorta and illiac arteries. ${ }^{1}$ It presents with the symptom complex of absent femoral pulses, intermittent claudication, gluteal pain and impotence. Intermittent claudication refers to muscle cramps in the legs which are reproducible by exercise. The exercise distance at which claudication develops is quite constant. It starts with the feet and proceeds to involve the buttocks. The chronic nature of the occlusive process promotes rich collateral blood supply to the lower extremity so that limbthreatening ischemia is uncommon and patient may be asymptomatic. However an acute occlusion may be associated with considerable morbidity and mortality. Conservative treatment includes risk factor modification, exercise, antiplatelet drugs, statin therapy and drugs like 
cilostazol which is an antiplatelet drug and a vasodilator. Severe disease usually requires angioplasty or surgical bypass.

\section{Case Report}

Our patient was a 51 year old diabetic female who presented with a history of severe pain in the back for the past 5 days which radiated down the right lower limb and to a lesser extent down the left side. It was accompanied by an intense sensation of pins and needles. Additionally there was numbness and burning sensation in the feet. Straight leg raising was negative. Power was normal. Ankle jerk was absent on both sides. Glove and stocking anaesthesia suggestive of diabetic polyneuropathy was present. The distal lower limb pulses were found to be absent. The feet were dry but not cold or blue. MRI of the lumbosacral spine showed that the lumber curvature was straightened. L4- L5 and L5- S1 intervertebral discs showed a diffuse bulge with narrowing of neural foramina and evidence of root compression. (Fig 1) There was bilateral ligamentum flavum hypertrophy with mild narrowing of spinal canal up to $8.2 \mathrm{~mm}$. She was treated conservatively for degenerative disc disease but did not improve and was reviewed. The odd point about the pain was that it appeared on walking and subsided with rest. Severe paraesthesias developed as she tried to stand up and she could barely walk. The patient also admitted to a longstanding history of intermittent claudication which began in the toes and feet and ascended to the buttocks. But she could walk comfortably for 3-4 kilometers. Suspecting a vascular disease a doppler study was done. Aorta and bilateral lower limb doppler showed monophasic tardus parvus flow in common femoral artery (CFA), superficial femoral artery (SFA), popliteal, anterior tibial, posterior tibial and dorsalis pedis artery in both lower limbs. CT angiography revealed extensive atherosclerotic thickening and calcified plaques in distal descending thoracic and abdominal aorta and its illiac branches. There was complete occlusion of infrarenal aorta $2.4 \mathrm{~cm}$ distal to renal arteries (Fig 2,3) and bilateral common illiac arteries by a hypodense thrombus and calcified plaques with reformation of bilateral illiac arteries by collaterals from epigastric and superior mesenteric vessels. She was given cilostazol, vasodilators, antiplatelet drugs and medication for neuropathy after which she made dramatic improvement. By the next day the patient was able to walk normally. Paraesthesias had disappeared although the burning in the feet persisted. She was advised to see a vascular surgeon for assessment of her PVD but she refused and opted for ayurvedic treatment along with life style and diet management .The burning feet also had also disappeared completely after 15 days.

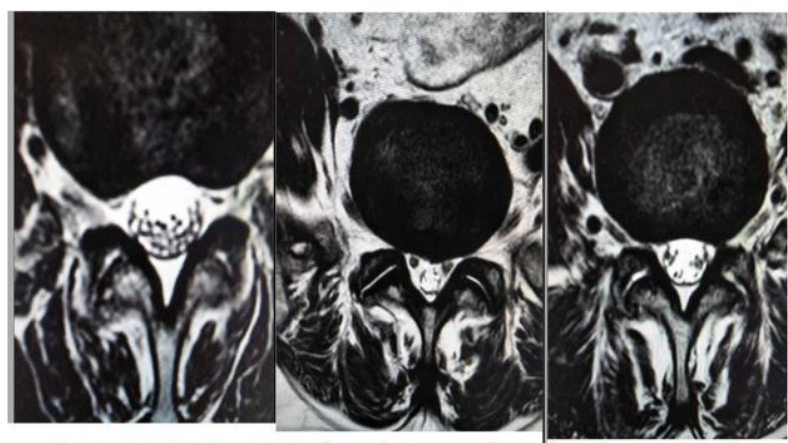

Fig $12 D$ MRI at $L_{3} L_{4}$ level normal and $L_{4} L_{5}$ and $L_{5} S_{1}$ showing forminal narrowing with compression of nerve roots bilaterally

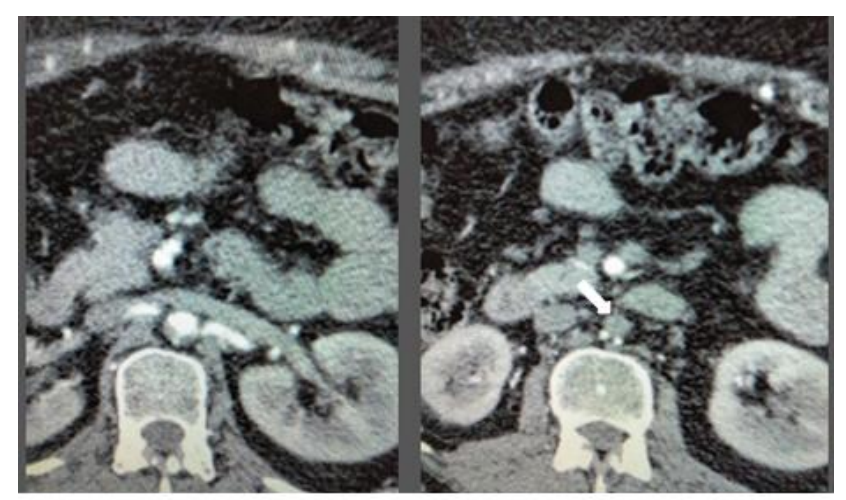

Fig 2 Occlusion of infrarenal aorta 


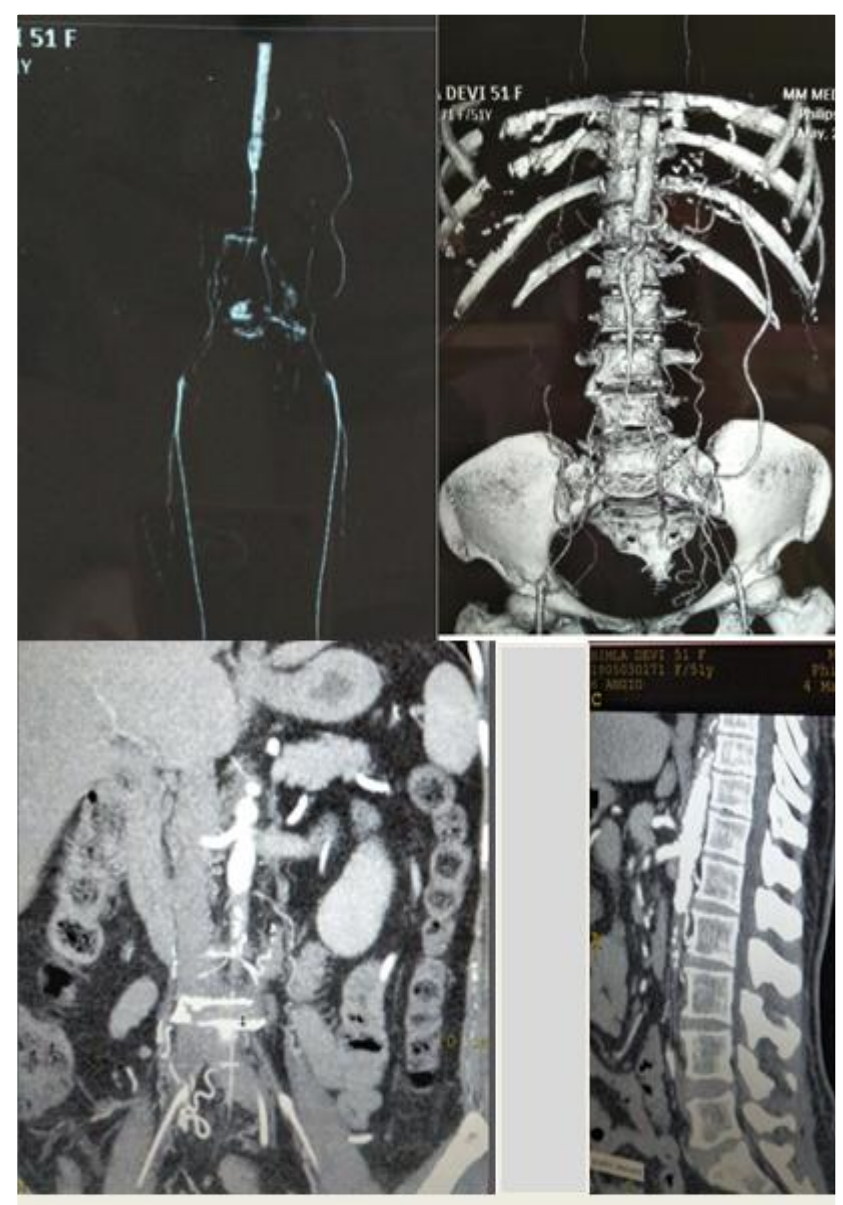

Fig 3 Occlusion of infrarenal aorta

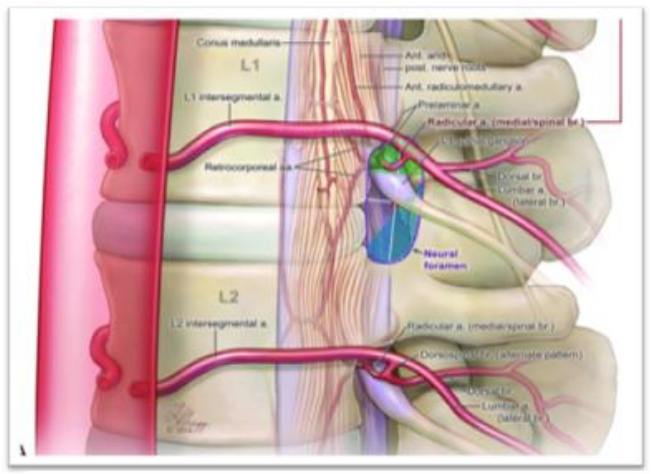

Fig 4 Blood supply to the nerve roots from aorta

\section{Discussion}

The patient had a complete occlusion of the infrarenal aorta with intermittent claudication but had not sought medical help till she developed the sciatica like symptoms due to degenerative disc disease which overshadowed the vascular disease. The symptoms in the disc disease usually improve on standing and may take a week or more to subside. But our patient worsened on exercise and made a dramatic recovery with medication to improve circulation. A possible hypothetical explanation could be that the symptoms due to mild foraminal and canal stenosis were aggravated by ischemia of the roots on exertion since there was prior vascular insufficiency due to the obstructive disease of the aorta which supplies the radicular vessels through its lumber branches. (Fig 4) This theory is supported by some reports which attribute radicular symptoms associated with degenerative disease of the lumbar spine to a combination of mechanical nerve root compression and resultant circulatory disturbance. Park et al postulated that amidst the dual blood supply of the roots there is a "watershed area," which could be responsible for the neuroischemic manifestations of compressive phenomenon involving the lumbar nerve roots. ${ }^{2}$ Reduced blood flow in the spinal nerve roots has been demonstrated during exercise. ${ }^{3}$ The oxygen tension in the interstices of a nerve trunk will be lowered during exercise if a simultaneous increase in the blood supply through the vasa nervorum is prevented as in our case due to the aortoilliac disease. ${ }^{4}$

There are other cases where the leriche syndrome presented with symptoms of a neurological disorder due to the ischemia of neural structures including spinal cord causing paraplegia due to reduced blood flow through artery of Adamkiewicz's. ${ }^{5}$ There are also 2 case reports of Leriche syndrome presenting as sciatic neuropathy /sciatica. ${ }^{6,7}$ In our case the presentation was a radiculopathy with elements of neurogenic and vascular claudication which might be linked as the key factor in both appears to be decreased blood supply especially on exercise.

The comparatively benign course in our patient is due to the development of collaterals .Diet and life style modification may alter the course of the disease for the better which may not always be a chronically progressive one which inevitably terminates in gangrene or death. ${ }^{8}$ 


\section{Conclusion}

This case serves as a reminder of the importance of a complete vascular evaluation in a case of diabetic who presents with symptoms of neuropathy particularly associated with walking.

\section{References}

1. Leriche, R; Morel, A (February 1948). "The Syndrome of Thrombotic Obliteration of the Aortic Bifurcation". Annals of Surgery. 127 (2): 193-206.

2. Parke WW, Watanabe R. The Intrinsic vasculature of the lumbosacral spinal nerve roots. Spine 1985; 10:508-515

3. Evans JG. Neurogenic intermittent claudication. Brit. med. J., 1964, 2, 985987

4. Blau, J. N., and Logue, V. (1961). Lancet, 1,1081

5. Akhaddar A, Eljebbouri B, Saouab R, Boucetta M. Acute paraplegia revealing Leriche syndrome. Intern Med. 2012;51:981-982

6. Yoon DH, Cho H, Seol SJ, Kim T.Right Calf Claudication Revealing Leriche Syndrome Presenting as Right Sciatic Neuropathy Ann 2014.Rehabil Medv $38(1)$;

7. Wang YC, Chiu YS, Yeh CH. Leriche's syndrome presenting as sciatica. Ann Vasc Surg. 2010;24:694.e1-694.e3

8. Victor G. Dewolfe, Fay A.et al Intermittent Claudication of the Hip and the Syndrome of Chronic Aorto-Iliac Thrombosis; Circulation 1954; no 1. 